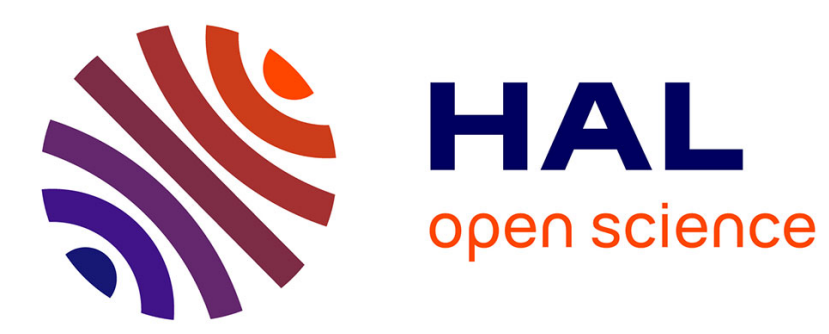

\title{
On the stability of large-scale streaks in turbulent Couette and Poiseulle flows
}

Junho Park, Yongyun Hwang, Carlo Cossu

\section{To cite this version:}

Junho Park, Yongyun Hwang, Carlo Cossu. On the stability of large-scale streaks in turbulent Couette and Poiseulle flows. Comptes Rendus Mécanique, 2011, vol. 339, pp. 1-5. 10.1016/j.crme.2010.10.012 . hal-00915603

\section{HAL Id: hal-00915603 \\ https://hal.science/hal-00915603}

Submitted on 9 Dec 2013

HAL is a multi-disciplinary open access archive for the deposit and dissemination of scientific research documents, whether they are published or not. The documents may come from teaching and research institutions in France or abroad, or from public or private research centers.
L'archive ouverte pluridisciplinaire HAL, est destinée au dépôt et à la diffusion de documents scientifiques de niveau recherche, publiés ou non, émanant des établissements d'enseignement et de recherche français ou étrangers, des laboratoires publics ou privés. 


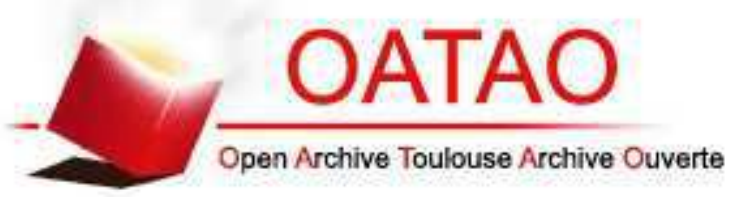

\section{Open Archive TOULOUSE Archive Ouverte (OATAO)}

OATAO is an open access repository that collects the work of Toulouse researchers and makes it freely available over the web where possible.

This is an author-deposited version published in : http://oatao.univ-toulouse.fr/ Eprints ID : 5426

To link to this article : DOI:10.1016/j.crme.2010.10.012

URL : http://dx.doi.org/10.1016/j.crme.2010.10.012

To cite this version : Park, Junho and Hwang, Yongyun and Cossu, Carlo. On the stability of largescale streaks in turbulent Couette and Poiseulle flows. (2011) Comptes Rendus Mécanique, vol. 339 $\left(n^{\circ} 1\right)$. pp. 1-5. ISSN 1631-0721

Any correspondance concerning this service should be sent to the repository administrator: staff-oatao@ listes-diff.inp-toulouse.fr 


\title{
On the stability of large-scale streaks in turbulent Couette and Poiseulle flows
}

\section{Sur la stabilité des streaks à grande échelle dans les écoulements de Couette et Poiseuille turbulents}

\author{
Junho Park ${ }^{\mathrm{a}}$, Yongyun Hwang ${ }^{\mathrm{a}, *}$, Carlo Cossu ${ }^{\mathrm{b}, \mathrm{c}}$ \\ a Laboratoire d'hydrodynamique (LadHyX), École polytechnique, 91128 Palaiseau cedex, France \\ b CNRS - Institut de mécanique des fluides de Toulouse (IMFT), allée du Pr. Camille-Soula, 31400 Toulouse, France \\ c Département de mécanique, École polytechnique, 91128 Palaiseau cedex, France
}

Keywords:

Instability

Secondary instability

Large-scale streaks

Turbulent flow

Mots-clés:

Instabilité

Instabilité secondaire

Streaks à grande échelle

Écoulements turbulent

\begin{abstract}
A B S T R A C T
The linear secondary stability of large-scale optimal streaks in turbulent Couette flow at $R e_{\tau}=52$ and Poiseulle flow at $\operatorname{Re}_{\tau}=300$ is investigated. The streaks are computed by solving the nonlinear two-dimensional Reynolds-averaged Navier-Stokes equations using an eddy-viscosity model. Optimal initial conditions leading the largest linear transient growth are used, and as the amplitude of the initial vortices increases, the amplitude of streaks gradually increases. Instabilities of the streaks appear when their amplitude exceeds approximately $18 \%$ of the velocity difference between walls in turbulent Couette flow and $21 \%$ of the centerline velocity in turbulent Poiseuille flow. When the amplitude of the streaks is sufficiently large, the instabilities attain significant growth rates in a finite range of streamwise wavenumbers that shows good agreement with the typical streamwise wavenumbers of the large-scale motions in the outer region.
\end{abstract}

\section{R É S U M É}

L'instabilité linéaire secondaire des streaks à grande échelle est étudiée dans les écoulements de Couette turbulent à $\operatorname{Re}_{\tau}=52$ et Poiseuille turbulent à $\operatorname{Re}_{\tau}=300$. Les streaks sont calculés en résolvant les équations de Navier-Stokes moyennées selon Reynolds en utilisant un modèle de viscosité turbulente. Les conditions initiales optimales, induisant la plus grande croissance transitoire, sont utilisées; quand l'amplitude des tourbillons optimaux initiaux est augmentée, l'amplitude des streaks augmente aussi. Les streaks deviennent instables quand leur amplitude est supérieure à environ $18 \%$ de la différence de vitesse entre les deux parois dans l'écoulement de Couette turbulent et $21 \%$ de la vitesse au centre du canal dans l'écoulement de Poiseuille turbulent. Quand l'amplitude des streaks est suffisamment élevée les instabilités atteignent des taux d'amplification significatifs dans une bande de longueurs d'onde qui est en bon accord avec les longueurs d'onde typiques observées dans les région externe.

\footnotetext{
* Corresponding author.

E-mail addresses: junho.park@ladhyx.polytechnique.fr (J. Park), yongyun@ladhyx.polytechnique.fr (Y. Hwang), carlo.cossu@imft.fr (C. Cossu).
} 


\section{Introduction}

Understanding the dynamics of coherent motions is a central issue in the research on wall-bounded turbulent flows. In the near-wall region, streaks, i.e. spanwise alternating patterns of high- and low-momentum regions with mean spacing about one hundred wall units, have been found as the most prominent feature [1]. These streaks sustain independently of the turbulent motions in the outer region [2], and the corresponding process has been understood as a cycle involving amplification of streaks by vortices, breakdown of the streaks via instability and the subsequent nonlinear process generating new vortices [3,4]. Streaky motions, however, have been also found in the outer region, and they carry a significant fraction of turbulent kinetic energy and Reynolds stress [5,6]. The origin of these large-scale streaky motions is not clear yet, but nonmodal stability theory has predicted that they can be significantly amplified by lift-up mechanism [7-9]. This encouraging result suggests that a self-sustaining process similar to the buffer-layer cycle presumably exists in the outer region, as confirmed by recent results [10]. However, streaks maximally amplified are found to be uniform in the streamwise direction, whereas the streaky motions observed in experiments have the finite streamwise wavelengths and meander with vortex packets (also called large-scale motions) coherently aligned to them $[5,6]$. Currently, there is no sound explanation for these features, and only recently it has been conjectured that the vortex packets may be related to the instability of large-scale streaks [5]. The goal of the present study is to analyze the stability of large-scale streaks and to seek a relationship between the streamwise wavelengths of the instability and the length-scales of the coherent structures in the outer region. In order to theoretically track this issue, we consider an eddy-viscosity model for the organized waves [11] successfully used to study the transient growth of large-scale streaks [7-9], and conduct a secondary stability analysis of the most amplified streaks in the turbulent Couette flow and in the turbulent Poiseulle flow.

\section{Background}

We consider the turbulent flow of an incompressible fluid with the density $\rho$ and kinematic viscosity $\nu$ in a channel with walls located at \pm . Here, the streamwise, wall-normal and spanwise directions are denoted as $x, y$ and $z$ respectively. For Couette flow, the upper and the lower walls move in opposite directions with the same velocity $U_{w}$. The Poiseulle flow is driven by a constant pressure gradient across the channel and has centerline velocity $U_{c l}$. For both cases, the equation for organized waves in perturbation form around the mean flow $\left(U_{i}=(U(y, z), 0,0)\right)$ is written as [11,7-9]

$$
\frac{\partial u_{i}}{\partial t}+u_{j} \frac{\partial U_{i}}{\partial x_{j}}+U_{j} \frac{\partial u_{i}}{\partial x_{j}}+u_{j} \frac{\partial u_{i}}{\partial x_{j}}=-\frac{1}{\rho} \frac{\partial p}{\partial x_{i}}+\frac{\partial}{\partial x_{j}}\left[\nu_{T}(y)\left(\frac{\partial u_{i}}{\partial x_{j}}+\frac{\partial u_{j}}{\partial x_{i}}\right)\right]
$$

Here, $u_{i}=(u, v, w)$ is the velocity of the organized wave and $v_{T}(y) \equiv v+v_{t}(y)$, where $v_{t}(y)$ is the turbulent eddy viscosity. The streaky base flow is computed using a mean profile $U(y)$ issued from DNS for the Couette flow as in [9] and the Reynolds-Tiederman profile for the Poiseulle flow as in [8]. $\nu_{T}(y)$ is the total eddy viscosity in equilibrium with $U(y)$ and the solutions are assumed uniform in the streamwise direction. Once the streaky base flow $u_{s}(y, z)$ is computed, the secondary base flow is defined as $U_{b}(y, z) \equiv U(y)+u_{s}(y, z)$. The stability of $U_{b}(y, z)$ is then studied by linearizing (1) with the secondary perturbations $u_{i}^{\prime}(x, y, z, t)$. Under the assumption that the base flow $U_{b}(y, z)$ is periodic in the spanwise direction, the Floquet theory allows the linearized equation to have the following normal-mode solution:

$$
u_{i}^{\prime}(x, y, z, t)=e^{i \alpha x-i \omega t} \sum_{n=-\infty}^{\infty} \hat{u}_{i}^{n}(y) e^{i(n+\epsilon) \beta_{0} z}+c . c .
$$

where $\alpha$ is the streamwise wavenumber, $\omega$ the complex frequency, $\beta_{0}$ the spanwise wavenumber related to the fundamental period of the base flow, and $0 \leqslant \epsilon \leqslant 1 / 2$ is the detuning parameter. According to the values of $\epsilon$, the solutions (2) are classified into 'fundamental' $(\epsilon=0)$ and 'subharmonic' $(\epsilon=1 / 2)$ modes. Also, the modes with even and odd symmetries about base flows are called 'sinuous' and 'varicose' respectively. For further details on the classification of the modes, the readers are referred to [12]. In the present study, we focus only on the fundamental sinuous mode, found as the most unstable one for all the cases considered.

Eq. (1) is discretized using Chebyshev polynomials and Fourier series in the wall-normal and spanwise directions respectively. The time integration used to compute $U_{b}(y, z)$ is conducted using the Runge-Kutta third-order method. For the Floquet analysis, the same spatial discretization is applied to the linear operators. The resulting numerical eigenvalue problem is then solved using the implicitly restarted Arnoldi method (for further details, see [12]). All the computations here are carried out with $N_{y} \times N_{z}=65 \times 32$.

\section{Results}

We consider a turbulent Couette flow at $R e_{\tau}=52$ and a Poiseulle flow at $R e_{\tau}=300$. The computation of the streaky base flows is carried out by using the optimal initial conditions, that consist of pairs of the counter-rotating streamwise vortices computed in [8,9] (see also Fig. 2). The spanwise spacing is chosen as $\lambda_{z}=4 h\left(\beta_{0} h=\pi / 2\right)$, which is very near the optimal value $[8,9]$. The spanwise size of computational box is set to as $L_{z}=\lambda_{z}$, so that a single pair of optimal initial 

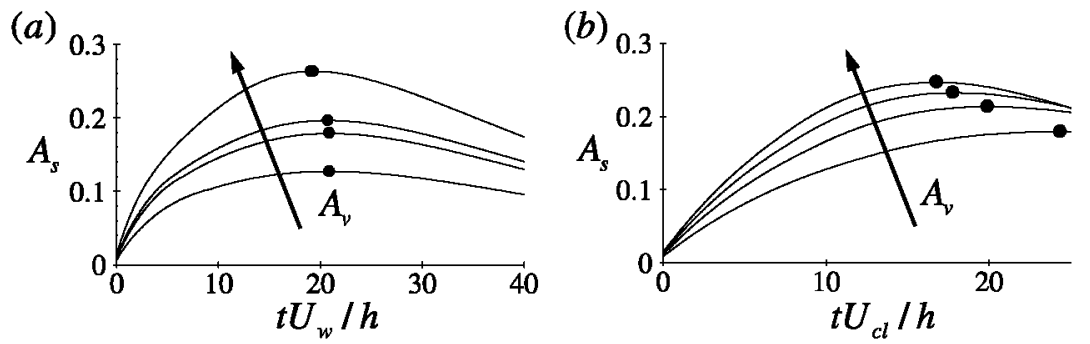

Fig. 1. Evolution of the streak amplitude in time: (a) Couette flow with $A_{v}=0.08,0.11,0.12,0.16$; (b) Poiseulle flow with $A_{v}=0.06,0.078,0.09,0.1$. Here, - denotes the maximum of $A_{s}$ at $t=t_{\max }$.
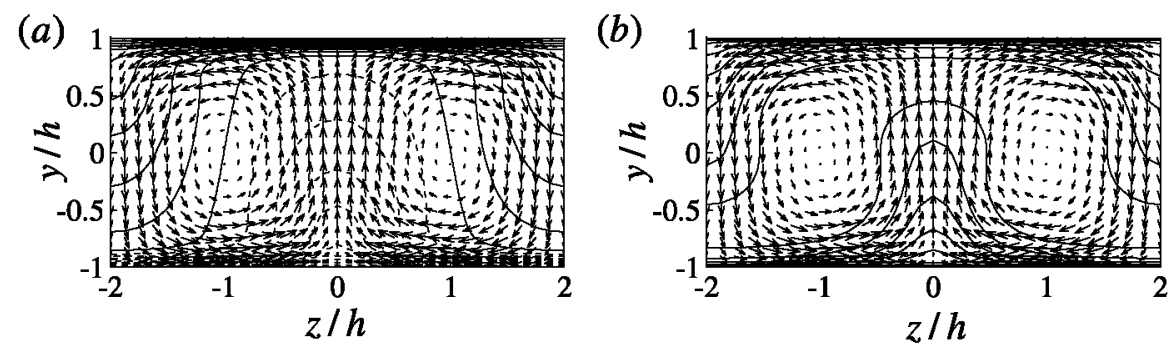

Fig. 2. Cross-stream $(y-z)$ view of streaky base flow extracted at $t=t_{\max }$ and optimal initial vortices: (a) Couette flow with $A_{5}=26 \%$ and (b) Poiseulle flow with $A_{s}=25 \%$. Here, the solid and dashed contour lines respectively denote positive and negative parts of the streaky base flow with the increment 0.1 of its maximum, and the vectors represent the initial vortices.
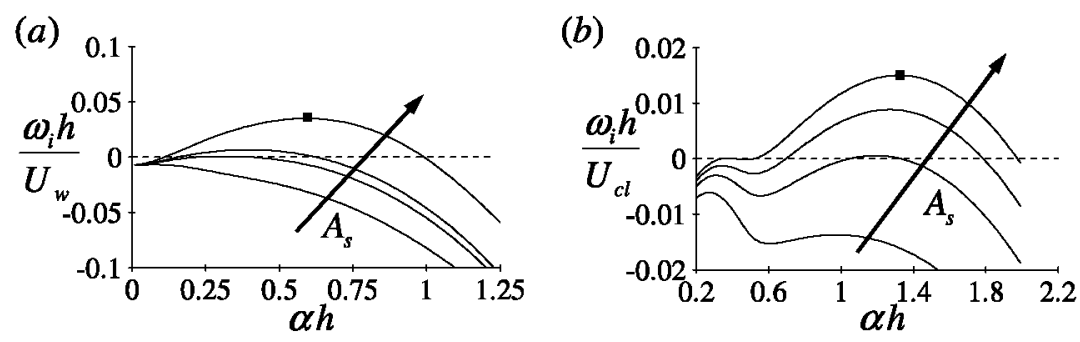

Fig. 3. Growth rate of fundamental sinuous mode with the streamwise wavenumber $\alpha$ for streaky base flows corresponding to • in Fig. 1: (a) Couette flow with $A_{s}=13,18,20,26 \%$; (b) Poiseulle flow with $A_{s}=18,21,23,25 \%$. Here, $\square$ indicates the location of maximum $\omega_{i}$ of the largest amplitude of streaks considered.

vortices is driven. The amplitude of the initial vortices is defined as $A_{v}=\left[(2 / V) \int_{V}\left(u^{2}+v^{2}+w^{2}\right) d V\right]^{1 / 2}$. The amplitude of the streaks induced by these vortices is defined [14] as

$$
A_{s}=\frac{\left[\max _{y, z} \Delta U(y, z)-\min _{y, z} \Delta U(y, z)\right]}{2 U_{\text {ref }}}
$$

where $\Delta U(y, z) \equiv u_{s}(y, z)$. Here, $U_{\text {ref }}=2 U_{w}$ and $U_{\text {ref }}=U_{c l}$ for Couette and Poiseulle flows respectively.

Fig. 1 shows the temporal evolution of the streak amplitude for several amplitudes $A_{\nu}$ of the optimal initial vortices. Both Couette and Poiseulle flows exhibit large transient amplifications of the streaks through the coherent lift-up effect. As $A_{v}$ increases, the amplitude of the streaks also increases. However, the ratio of the amplification slightly decays with increasing $A_{v}$, and the time for $A_{s}$ to reach its maximum ( $\left.t_{\max }\right)$ also becomes shorter. Typical cross-stream views of the initial vortices and streaks at $t=t_{\max }$ are reported in Fig. 2 . It is seen that the low-momentum regions where the fluid is ejected from the wall by the vortices $((y>0, z \simeq \pm 2 h)$ and $(y<0, z \simeq 0)$ in Fig. 2) are narrow and intense, and this tendency generally becomes stronger for larger streak amplitudes.

The Floquet stability analysis is performed for the fundamental modes using the streaky base flows extracted at $t=t_{\max }$. Fig. 3 shows the growth rate $\omega_{i}$ of sinuous modes versus the streamwise wavenumber $\alpha$ for several $A_{s}$. Both Couette and Poiseulle flows are stable for sufficiently low amplitudes of the streaks $\left(\omega_{i}<0\right)$. As the amplitude of streak $A_{s}$ increases, the growth rate gradually increases. Then instability begins to appear $\left(\omega_{i}>0\right)$ when $A_{s}$ reaches the value: $A_{s, c}=18 \%$ at $\alpha_{c} h=0.3$ for Couette flow (Fig. 3a) and $A_{s, c}=21 \%$ at $\alpha_{c} h=1.2$ for Poiseulle flow (Fig. 3b). When $A_{s}>A_{s, c}$, a finite interval of the streamwise wavenumbers is unstable with maximum growth at $\alpha_{\max } h \simeq 0.6$ in Couette flow (Fig. 3a) and $\alpha_{\max } h \simeq 1.4$ in Poiseulle flow (Fig. 3b). We have verified that varicose modes are less unstable than sinuous modes in this range of $A_{v}-A_{s}$.

Fig. 4 shows the spanwise velocity component $w$ of the streak-instability eigenfunction and the line where the mean velocity is the same as the phase speed $c_{r}$ of instability wave ( $c_{r}=0$ in Couette flow while $c_{r}=0.87 U_{c l}$ in Poiseulle flow). The w-component of the eigenfunction is concentrated in the lifted low-momentum region for both Couette and Poiseulle 

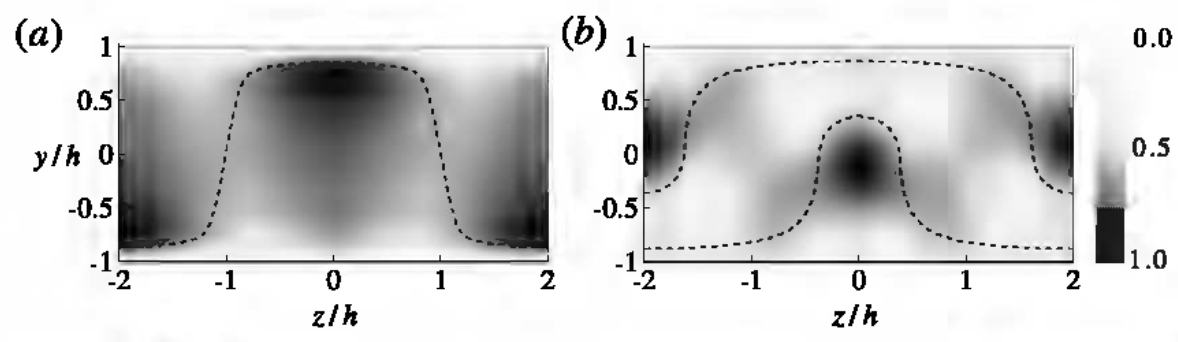

Fig. 4. Cross-stream $(y-z)$ view of the absolute value of the spanwise velocity eigenfunction corresponding to $\mathbf{a}$ in Fig. 3: (a) Couette flow; (b) Poiseulle flow. Here, the contour is normalized by its maximum and the thick dashed line denotes the velocity of streaky base flow which is the same with the phase speed of the streak instability.

flows. In Couette flow the amplitude of the eigenfunction is appreciable even close to the opposite wall, whereas in Poiseulle flow it is mainly concentrated on the center of the channel, which is reminiscent of the unstable modes found in the laminar case.

\section{Discussion}

We have investigated the instability of finite amplitude streaks in the framework of an eddy viscosity of turbulent Couette and Poiseulle flows. The nature of the streak instability we find bears same similarity the one observed in the laminar flows: the instability appears for the high amplitude streaks and it is dominated by the fundamental sinuous mode. An interesting point is that the critical streak amplitudes in the present model are lower than those in laminar flows: for example, our own computations in laminar Couette and Poiseulle flows at $R e_{h}=500$ give $A_{s, c} \simeq 23 \%$ and $A_{s, c} \simeq 28 \%$ respectively (see also [13]), whereas for the turbulent Couette and Poiseulle flows it is found $A_{s, c} \simeq 18 \%$ and $A_{s, c} \simeq 21 \%$ respectively at $R e_{\tau}=52$ and $R e_{\tau}=300$. However, this does not mean that the streak instability in turbulent flows can be more easily triggered than in laminar flows because as the optimal turbulent transient growth is smaller than the laminar one [8,9], initial vortices in turbulent flows should be more energetic to drive streak instability than those in laminar flows. For example, in the laminar Poiseulle flow, $A_{v, c} \sim O\left(10^{-3}\right)$ is required to trigger streak instability [13], but the amplitude of initial vortices in the present model of Poiseulle flow is almost two order of magnitude larger (i.e. $A_{\mathbf{v}, c} \simeq 0.08$ ).

In the outer region of the turbulent Poiseulle flow, the streamwise length scale of the large-scale motions has been associated with the peak near the high wavenumber boundary of the $\alpha^{-1}$ regime in the spectra of the outer region at $\alpha h=$ $1 \sim 2\left(\lambda_{x}=3.1 \sim 6.3 h\right)$, and that is clearly visible for $0<|y|<0.6 h[15,16]$. The range of unstable streamwise wavenumbers in the present study is also obtained at $\alpha h=0.8 \sim 1.8\left(\lambda_{x}=3.5 \sim 7.9 h\right.$; see Fig. 3b), showing good agreement with the location of the peak in the streamwise spectra. Moreover, the eigenfunctions obtained here have significant values in $0<$ $|y|<0.6 \mathrm{~h}$, also comparable to the spectra. For the turbulent Couette flow, the streamwise wavenumber having the maximum growth rate ( $\alpha h \simeq 0.6 ; \lambda_{x} \simeq 10.5 h$; see Fig. 3a) also corresponds well to the location of the peak in the streamwise spectra at the channel center $\left(\alpha h \simeq 0.63 ; \lambda_{x} \simeq 10 h\right.$ ) [17]. This good correlation in the length-scale comparison implies that the largescale motion could be formed by instability of much longer streaky motions in the outer region. However, as the Reynolds numbers considered here are fairly low, this conclusion remains to be confirmed at larger Re. Also, alternative scenario based on the secondary transient growth as in [18] may also be relevant, and is currently under active investigation.

\section{Acknowledgements}

J.P. acknowledges funding from the French Ministry of Foreign Affairs through a Blaise-Pascal Scholarship. Y.H. acknowledges funding from Ėcole polytechnique through a Gaspard-Monge Scholarship. The partial support of DGA is also gratefully acknowledged.

\section{References}

[1] S.J. Kline, W.C. Reynolds, F.A. Scturaub, P.W. Runstadler, The structure of turbulent boundary layers, J. Fluid Mech, 30 (1967) 741-773.

[2] J. Jimenez, A. Pinelli, The zutonomous cycle of near-wall turbulence, J. Fluid Mech. 389 (1999) 335-359.

[3] J.M. Hamilton, J. Kim, F. Waleffe, Regeneration mechanisms of near-wall turbulence structures, J. Fluid Mech. 287 (1995) 317-348.

[4] F. Waleffe, On a self-sustaining process in shear flows, Phys. Fluids 9 (1997) 883-900.

[5] M. Guala, S.E. Hommema, RJ. Adrian, Large-scale and very-large-scale motions in turbulent pipe flow, J. Fluid Mech. 554 (2006) $521-541$.

[6] N. Hutchins, I. Marusic, Evidence of very long meandering features in the logarithmic region of turbulent boundary layers, J. Fluid Mech. 579 (2007) $1-28$.

[7] J.C. del Alamo, J. Jiménez, Linear energy amplification in turbulent channels, J. Fluid Mech. 559 (2006) 205-213.

[8] G. Pujals, M. García-Villalba, C. Cossu, S. Depardon, A note on optimal transient growth in turbulent channel flows, Phys. Fluids 21 (015109) (2009) 1-6.

[9] Y. Hwang, C. Cossu, Amplification of coherent streaks in the turbulent Couette flow: an input-output analysis at low Reynolds number, J. Fuid Mech. 643 (2010) 333-348.

[10] Y. Hwang, C. Cossu, On a self-sustained process at large scales in the turbulent channel flow, Phys. Rev. Latt. 105 (2010) 044505.

[11] W.C. Reynolds, A.K.M.F. Hussain, The mechanics of an organized wave in turbulent shear flow. Part 3. Theoretical models and comparisons with experiments, J. Fluid Mech. 54 (1972) 263-288. 
[12] C. Cossu, L. Brandt, On Tollmien-Schlichting waves in streaky boundary layers, Eur. J. Mech./B Fluids 23 (2004) 815-833.

[13] S.C. Reddy, P.J. Schmid, J.S. Baggett, D.S. Henningson, On the stability of streamwise streaks and transition thresholds in plane channel flows, J. Fluid Mech. 365 (1998) 269-303.

[14] P. Andersson, L. Brandt, A. Bottaro, D. Henningson, On the breakdown of boundary layers streaks, J. Fluid Mech. 428 (2001) $29-60$.

[15] B.J. Balakumar, R. Adrian, Large- and very-large-scale motions in channel and boundary layer flows, Phil. Trans. R. Soc. A 365 (1852) (2001) 665-681.

[16] J.P. Monty, N. Hutchins, H.C.H. Ng, I. Marusic, M.S. Chong, A comparison of turbulent pipe, channel and boundary layer flows, J. Fluid Mech. 632 (2009) $431-442$.

[17] T. Tsukahara, H. Kawamura, K. Shingai, DNS of turbulent Couette flow with emphasis on the large-scale structure in the core region, Journal of Turbulence 7 (19) (2006).

[18] W. Schoppa, F. Hussain, Coherent structure generation in near-wall turbulence, J. Fluid Mech. 453 (2002) 57-108. 